\title{
Autocorrelation of scattered laser light for ultrasound-modulated optical tomography in dense turbid media
}

\author{
Hui Li and Lihong V. Wang
}

\begin{abstract}
Based on measurement of the intensity autocorrelation function, a new method to determine the modulation depth of scattered laser light modulated by an ultrasonic wave in turbid media was applied to ultrasound-modulated optical tomography. Good signal-to-noise ratios and high sensitivities were demonstrated. Images of double optically absorbing objects buried in a highly optically scattering gel sample were obtained. The contrast was more than $10 \%$, and the spatial resolution was approximately $2 \mathrm{~mm}$. (C) 2002 Optical Society of America
\end{abstract}

OCIS codes: $170.3880,030.6140,170.5270,170.7050,110.7170$.

The detection of tumors, based on the measurement of optical property differences between normal and abnormal tissues, has been a very active field of research in recent years. Among the hot topics in this area, ultrasound-modulated optical tomography that combines optical with ultrasonic techniques has demonstrated some promising results. Poor optical resolution, resulting from strong scattering, and small mechanical contrast between early-stage tumors and normal soft tissues are avoided. Another advantage of this hybrid method is that of the high contrast of light and the good localization of sound in tissues. With this technique, an ultrasonic wave is focused into a scattering medium to modulate light propagating through an acoustic beam. The modulated part of the detected light can be discriminated from the nonmodulated background light, and the origin of the modulated light can be directly derived from the position of the ultrasonic column inside the medium. The amount of modulated light relative to the total amount of light, i.e., the modulation depth $M$, can be used to identify the tissue's optical property at the

The authors are with the Optical Imaging Laboratory, Biomedical Engineering Program, Texas A\&M University, College Station, Texas 77843-3120. H. Li is also with the Department of Physics, Fujian Teachers University, Fujian 350007 China. L. V. Wang's e-mail address is LWang@tamu.edu.

Received 15 January 2002; revised manuscript received 9 April 2002.

0003-6935/02/224739-04\$15.00/0

(C) 2002 Optical Society of America ultrasonic beam if its ultrasonic difference is negligible.

Leutz and Maret, ${ }^{1}$ Mahan et al., ${ }^{2}$ and Wang ${ }^{3,4}$ studied the theoretical mechanisms of interaction between ultrasonic waves and light in turbid media. Leutz and Maret $^{1}$ modeled the incoherent mechanism based on the variations of the optical phase in response to ultrasound-induced displacements of scatterers, but their theory is valid only when the scattering mean free path is much greater than the acoustic wavelength. Mahan et al. ${ }^{2}$ modeled an incoherent mechanism based on the ultrasonic modulation of optical properties; however, this mechanism is too weak to produce experimentally observable results. Wang ${ }^{3,4}$ modeled two coherent mechanisms for the ultrasonic modulation of light in scattering media: one is based on the ultrasound-induced displacements of scatterers and the other is based on the ultrasound-induced variations of the refractive index.

In the experimental realm, there have been some major developments since the observation of ultrasonic-modulated optical signals by Marks et al. ${ }^{5}$ Wang et al. ${ }^{6}$ and Wang and Zhao ${ }^{7}$ first developed ultrasound-modulated optical tomography, then Wang and $\mathrm{Ku}^{8}$ employed a frequency-sweep technique to provide imaging resolution along the ultrasonic axis. Kempe et al. ${ }^{9}$ showed experimentally the transition of ultrasonic modulation from the ballistic to the scattered regimes. In addition to those singledetector schemes, Leveque et al..$^{10}$ employed parallel detection to improve the signal-to-noise $(\mathrm{S} / \mathrm{N})$ ratio by using a CCD camera. Yao and Wang ${ }^{11}$ further demonstrated this scheme with two-dimensional im- 
ages of biological tissues. By combining the parallel-detection and the frequency-swept techniques, Yao et al. ${ }^{12}$ also obtained two-dimensional images with controllable resolution along the acoustic axis. Recently, Lev et al. ${ }^{13}$ developed a reflection configuration.

Despite the above-mentioned developments, for the practical requirements of medical applications to be achieved, researchers continue endeavors to improve image quality and to increase the imaging depth. In this paper, we applied, for the first time to our knowledge, an intensity autocorrelation function of scattered light to obtain one-dimensional images of a dense tissuelike phantom by photon-counting techniques. Obviously, photon counting is superior, in terms of $\mathrm{S} / \mathrm{N}$ ratio and sensitivity, to processing the output of the photomultiplier tube (PMT) directly as a current. Although it is a single-detector scheme, one can increase the $\mathrm{S} / \mathrm{N}$ ratio to exceed the level of previous parallel detection by multiple averaging at the expense of time. Therefore, because of its high $\mathrm{S} / \mathrm{N}$ ratio, it offers the feasibility of detecting a deeper object in strong scattering media under the same condition of the incident light, which should, of course, be below the damage threshold for biological tissues. This new method falls into the family of dynamic light-scattering techniques known as a homodyne photon-correlation spectroscopy, but it is somewhat different from the general means because our goal is to characterize the periodical information at the ultrasonic frequency. For this reason, we directly derived the relation between the modulation depth of the scattered light and the intensity autocorrelation function, $G^{(2)}$, which was more direct than the field autocorrelation function, $G^{(1)}$, used by Leutz et al. ${ }^{1}$ By the way, there is a complicated relation between $G^{(2)}$ and $G^{(1)}$ in a multiple-scattering domain.

The temporal signal of detected scattered light can be represented as

$$
I_{s}(t)=I_{0}[1+M \cos (\Omega t+\phi)],
$$

where $I_{0}$ is the background intensity, $\Omega$ is the angular frequency of the ultrasonic wave, $M$ is the modulation depth related to the modulated ac signal that reflects the local optical and ultrasonic properties, and $\phi$ is the initial phase of the modulated signal.

The intensity autocorrelation function is obtained with

$$
G^{(2)}(\tau)=\left\langle I_{s}(t) I_{s}(t+\tau)\right\rangle=I_{0}^{2}\left[1+\left(M^{2} / 2\right) \cos (\Omega \tau)\right] .
$$

The normalized intensity autocorrelation function is written

$$
g^{(2)}(\tau)=1+\left(M^{2} / 2\right) \cos (\Omega \tau) .
$$

Equation (2) or (3) is the basis used to measure the modulation depth $M$, from which it can be noted that the modulation depth of $G^{(2)}$ is $M^{2} / 2$. The intensity autocorrelation function is independent of the initial

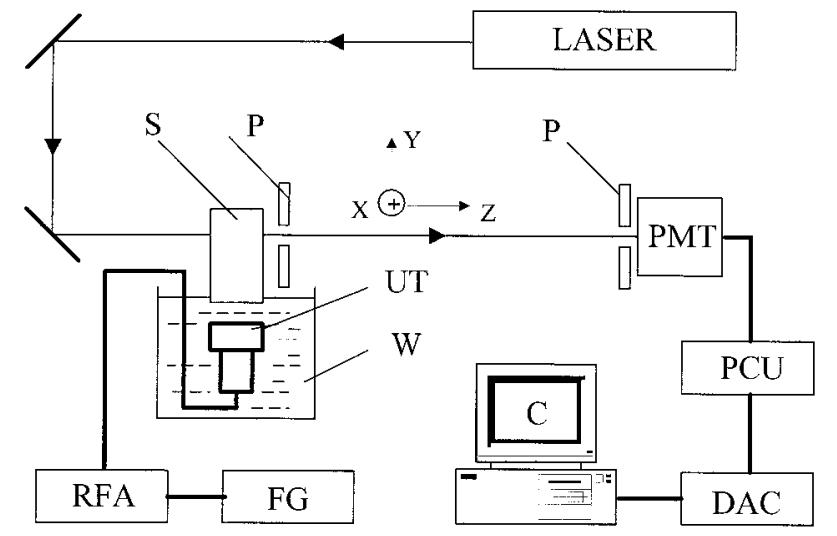

Fig. 1. Experimental setup: LASER, 532-nm laser with an output of $800 \mathrm{~mW}$; S, sample; W, water; UT, ultrasonic transducer; RFA, RF amplifier; FG, function generator; P, pinholes; PMT, photomultiplier tube; PCU, photon-counting unit; DAC, digital autocorrelator; and C, computer.

phase of the modulated signal $\phi$. Because we are interested only in detection of the autocorrelation function in a narrow bandwidth centered around $\Omega$, variations outside this band do not contribute much to the autocorrelation function.

The experimental setup is shown in Fig. 1 . The $X$ axis was perpendicular to both the acoustic and optical axes, noted as $Y$ and $Z$, respectively (see Fig. 2). A laser system (Verdi V5, Coherent, Inc., Santa Clara, California) was used as the light source. The laser beam of 532-nm wavelength, 3-mm diameter, and $800-\mathrm{mW}$ power was incident on the sample normally. For biological tissues, the laser beam can be expanded to avoid damage without affecting the ultrasonic modulation significantly. The coherence length of the laser beam was approximately $60 \mathrm{~m}$. Two pinholes separated by $350 \mathrm{~mm}$ were used; the aperture of the pinhole close to the sample was 0.5 $\mathrm{mm}$, and the other one was $2.0 \mathrm{~mm}$. The setup of the pinholes caused the detection area in the PMT to include approximately five speckles. Because the phases of speckles are uncorrelated, the more speckles collected by the PMT, the lower the modulation depth. Of course, there is a trade-off between the intensity of the detected light and the modulation depth. A focused ultrasonic transducer (V314, Panametrics, Inc., Waltham, Massachusetts; focal length, $2.54 \mathrm{~cm}$; waist size, $2 \mathrm{~mm}$ ) generated a $1-\mathrm{MHz}$ ultrasonic wave. The peak pressure at the focal spot was approximately $2 \times 10^{5} \mathrm{~Pa}$. The function generator (DS345, Stanford Research Systems, Inc., Sunnyvale, California) output was a $1-\mathrm{MHz}$ sinusoidal signal to the rf power amplifier, which excited the ultrasonic transducer.

The photon-counting unit (C3866, Hamamatsu Photonics K. K., Hamamatsu City, Japan) was used for converting the output of the PMT [R2949 Hamamatsu, supply voltage, $-850 \mathrm{~V}$; dark counts, $\sim 0.5 \mathrm{Kcps}$ (counts per second)] into $5-\mathrm{V}$ digital signals by means of an amplifier-discriminator system. The photon-counting unit can provide excellent lin- 
earity (no pulse overlapping) in the intensity range between 1 and $10^{7} \mathrm{cps}$. Combining the R2949 and the C3866, one can expect a dynamic light-intensity range from 500 to $10^{7} \mathrm{cps}$, i.e., from $2 \times 10^{-16}$ to $4 \times$ $10^{-12} \mathrm{~W}$ at a wavelength of $532 \mathrm{~nm}$. A photoncounting digital system (Flex2K-12 $\times 2$, Correlator. com) was used as a fast real-time, multiple- $\tau$ digital correlator, which calculated the autocorrelation function $g^{(2)}(\tau)$ in real time, covering delay times from a minimum sample time of $12.5 \mathrm{~ns}$ to $\sim 1 \mathrm{~h}$ with more than 288 data points. The sample time is doubled for every eight data points. The delay times were (1, $2, \ldots 16) \times 12.5 \mathrm{~ns},(9,10, \ldots 16) \times 25 \mathrm{~ns},(9$, $10, \ldots 16) \times 50 \mathrm{~ns}$, and so on. In other words, the system can provide the $1-\mathrm{MHz}$ modulation information of the $g^{(2)}(\tau)$ during a delay time ranging $\tau$ from 1 to $5 \mu \mathrm{s}$. We found that the dark counts of the PMT could be ignored as long as the detected light intensity reached approximately 1 Mcps by even one measurement. It was discovered that with photon counting and the correlation method, a modulated signal buried in the detected light that was as weak as $4 \times 10^{-13} \mathrm{~W}$ could be detected. This level of detection is at approximately the same magnitude of sensitivity as that resulting from measuring PMT current when the $\mathrm{S} / \mathrm{N}$ ratio is equal to 1 and the modulation depth is $\sim 10 \%$. However, the $\mathrm{S} / \mathrm{N}$ ratio based on photon counting and correlation can reach 10 or more, which equals the detected intensity $\times$ modulation depth/dark counts (e.g., $10^{6} \mathrm{cps} \times 10 \% /$ $10^{4} \mathrm{cps} \approx 10$ ) under the same conditions. Consequently, because of its high $\mathrm{S} / \mathrm{N}$ ratio, this method offers promise for detecting tumors in denser scattering media or thicker biological tissues than other methods in ultrasound-modulated optical tomography.

Three tissuelike samples were used in our experiments. Each of the samples, in which two objects were buried at different positions along the $Z$ axis, was a gel containing milk as the scatterers. The reduced scattering coefficient of the samples was $\sim 12$ $\mathrm{cm}^{-1}$ at $532 \mathrm{~nm}$, which was comparable with that of typical biological tissues in the red and near-infrared wavelength regions. The absorption coefficient of the gel was negligible compared with the reduced scattering coefficient. The objects were made from black-rubber tubes $(4.2 \mathrm{~mm}$ in diameter, $24 \mathrm{~mm}$ in length) filled with the same gel. A small amount of water was used to fill the gaps between the objects and the background gel to avoid air bubbles. The tubes were used because the gel core in the tubes had the same acoustic property as the background gel, but the shell of the tubes had complete optical absorption compared with the surrounding gel. The axes of the tubes were parallel with the acoustic axis. Because of the thin shell $(\sim 0.2 \mathrm{~mm})$ and the large diameter $(\sim 4.2 \mathrm{~mm})$ of the tubes compared with the acoustic wavelength $(1.5 \mathrm{~mm})$ and the ultrasound waist size $(\sim 2 \mathrm{~mm})$, the acoustical interference of the tubes is negligible. The gel sample was partially immersed in water for good acoustic coupling.

The positions of the buried objects and the three

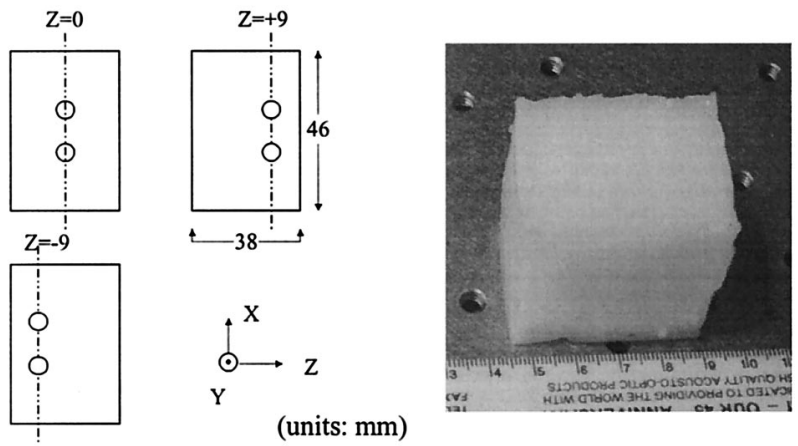

Fig. 2. Configurations of the absorbing objects and photograph of the phantom. The $Z$ axis is along the optical axis that is normal to the sample surface. The $Y$ axis is along the acoustic axis pointing to the sample. The $X$ axis is the direction of scanning, perpendicular to both the acoustic and the optical axes, $Y$ and $Z$, respectively.

scanning lines of the ultrasonic transducer are indicated in Fig. 2. A photograph of the gel sample is also given in the figure. In the experiments, the laser beam, the transducer, and the detector were kept fixed, and the sample was scanned with a translation stage.

Figure 3 shows the intensity of the detected scattered light (filled symbols) and the modulation depth (open symbols) at various depths in the samples. It can be seen that there is no information for distinguishing the two absorption objects apart in the curves of the intensity of the detected scattered light. The curves of the modulation depth show the corresponding object positions with errors in the modulation depth of approximately 5\%, which can be reduced if additional measurements are made. We can clearly see that the image contrast is more than $10 \%$. The width of each dip corresponding to the objects is approximately $8 \mathrm{~mm}$, which is in good agreement with the size of the objects convolved with the resolution because the signal is, in fact, the convolution between the object $(4.2 \mathrm{~mm}$ in diameter) and the ultrasonic waist ( $2 \mathrm{~mm}$ in diameter). It can be seen

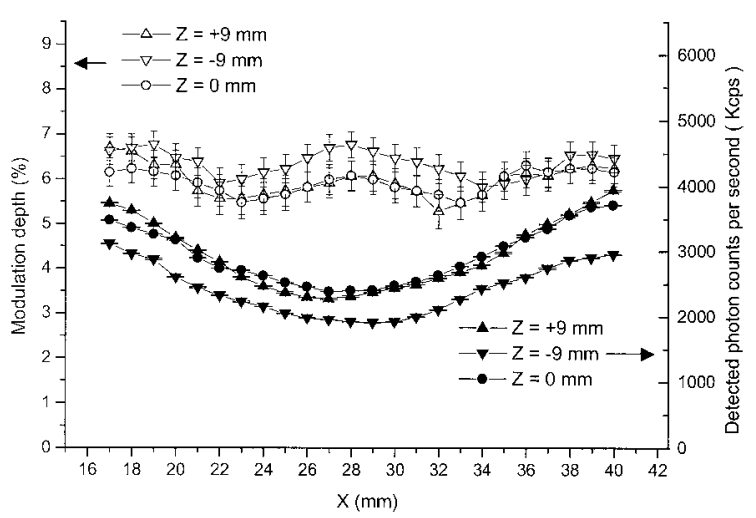

Fig. 3. Detected scattered light intensity in photon counts per second (filled symbols) and the modulation depth (open symbols) versus the scanning position $(X)$ at various depths $(Z)$ in the gel samples. 
that the modulation depth at $Z=-9 \mathrm{~mm}$ is higher than at $Z=0$ and $+9 \mathrm{~mm}$, whereas the intensity of the detected light at $Z=-9 \mathrm{~mm}$ is lower than at $Z=$ 0 and $+9 \mathrm{~mm}$. This result can be explained by the distribution of the light fluence rate in the sample and by the perturbation of the ultrasonic column as well as the two buried objects to the light that ultimately reaches the detector. The perturbation is greater at positions closer to the incident light source where the light has more opportunities to pass through the ultrasonic column or the buried objects. Larger modulation depths and smaller intensities are therefore obtained at these positions. Yao and Wang ${ }^{11}$ achieved a similar result for the modulation depth in their Monte Carlo simulation in which the modulation signal decreased when the ultrasonic column was moved away from the light source. The perturbation plateaus at positions far enough from the source where the light distribution is nearly uniform vertically; consequently, the opportunities for light to pass though the ultrasonic column or the buried objects are fairly constant. As a result, the signals at $Z=0$ and $+9 \mathrm{~mm}$ show little difference. It should be emphasized that the thickness of the tissuelike samples was $38 \mathrm{~mm}$, which approaches the thickness of practical medical interest.

In conclusion, a new method to measure modulation depth, which was based on the measurement of the intensity autocorrelation function, was presented. The method was applied in ultrasoundmodulated optical tomography, which demonstrated that the method has advantages in providing a good $\mathrm{S} / \mathrm{N}$ ratio and high sensitivity. Light intensity as low as $10^{-13} \mathrm{~W}$ may be detected. The variation of the modulation depth along the direction of the incident light was found to be consistent with the prediction of a Monte Carlo simulation published previously. ${ }^{11}$ It was demonstrated that $4.2-\mathrm{mm}$ diameter, optically absorbing objects, which were buried in different depths in the 38-mm tissuelike media, were clearly imaged with a transmission scheme. An image contrast of $10 \%$ and a spatial resolution of approximately $2 \mathrm{~mm}$ were obtained.

The resolution is determined mainly by the ultrasound waist size and can be improved by reducing the waist size at the expense of modulation depth and $\mathrm{S} / \mathrm{N}$ ratio.
This project was sponsored in part by the National Institutes of Health grant R01 CA71980; National Science Foundation grant BES-9734491; and Texas Higher Education Coordinating Board grant 0005120123-1999. H. Li is a visiting scholar at Texas A\&M University sponsored by the China Scholarship Council.

\section{References}

1. W. Leutz and G. Maret, "Ultrasonic modulation of multiply scattered light," Physica B 204, 14-19 (1995).

2. G. D. Mahan, W. E. Engler, J. J. Tiemann, and E. Uzgiris, "Ultrasonic tagging of light: Theory," Proc. Natl. Acad. Sci. USA 95, 14015-14019 (1998).

3. L.-H. V. Wang, "Mechanisms of ultrasonic modulation of multiply scattered coherent light: a Monte Carlo model," Opt. Lett. 26, 1191-1194 (2001).

4. L.-H. V. Wang, "Mechanisms of ultrasonic modulation of multiply scattered coherent light: an analytic model," Phys. Rev. Lett. 87(4), 043903, 1-4 (2001).

5. F. A. Marks, H. W. Tomlinson, and G. W. Brooksby, "Comprehensive approach to breast cancer detection using light: photon localization by ultrasound modulation and tissue characterization by spectral discrimination," in Photon Migration and Imaging in Random Media and Tissue, B. Chance and R. R. Alfano, eds., Proc. SPIE 1888, 500-510 (1993).

6. L.-H. Wang, S. L. Jacques, and X. Zhao, "Continuous-wave ultrasonic modulation of scattered laser light to image objects in turbid media," Opt. Lett. 20, 629-632 (1995).

7. L.-H. Wang and X. Zhao, "Ultrasound-modulated optical tomography of absorbing objects buried in dense tissuesimulating turbid media," Appl. Opt. 36, 7277-7282 (1997).

8. L.-H. Wang and G. Ku, "Frequency-swept ultrasoundmodulated optical tomography of scattering media," Opt. Lett. 23, 975-977 (1998).

9. M. Kempe, M. Larionov, D. Zaslavsky, and A. Z. Genack, "Acousto-optic tomography with multiple scattered light," J. Opt. Soc. Am. 14, 1151-1158 (1997).

10. S. Leveque, A. C. Boccara, M. Lebec, and H. Saint-Jalmes, "Ultrasonic tagging of photon paths in scattering media: parallel speckle modulation processing," Opt. Lett. 24, 181-183 (1999).

11. G. Yao and L.-H. V. Wang, "Theoretical and experimental studies of ultrasound-modulated optical tomography in biological tissue," Appl. Opt. 39, 659-664 (2000).

12. G. Yao, S.-L. Jiao, and L.-H. V. Wang, "Frequency-swept ultrasound-modulated optical tomography in biological tissue by use of parallel detection," Opt. Lett. 25, 734-736 (2000).

13. A. Lev, Z. Kotler, and B. G. Sfez, "Ultrasound tagged light imaging in turbid media in a reflectance geometry," Opt. Lett. 25, 378-380 (2000). 